\title{
Data-Driven Dynamic Neural Programming for Network Media Nonlinear Visual Communication Design
}

\author{
Jing Yang $\mathbb{D}^{1}$ and Xiaoman $\mathrm{Li}^{2}$ \\ ${ }^{1}$ Hunan First Normal University, Changsha 410205, Hunan, China \\ ${ }^{2}$ Shih Chien University, Taipei 222, Taiwan \\ Correspondence should be addressed to Jing Yang; msyyangjing@hnfnu.edu.cn
}

Received 6 December 2021; Revised 4 January 2022; Accepted 10 January 2022; Published 21 February 2022

Academic Editor: Gengxin Sun

Copyright (c) 2022 Jing Yang and Xiaoman Li. This is an open access article distributed under the Creative Commons Attribution License, which permits unrestricted use, distribution, and reproduction in any medium, provided the original work is properly cited.

\begin{abstract}
As network media data gradually enters the field of visual communication design as information, more scholars have begun to rationally study the relationship between visual communication and network media. This paper mainly studies how to use datadriven nonlinear dynamic neural programming method to realize visual communication design in network media environment. The study constructs a network media visual communication design system from three aspects: data-driven, design process realization, and system flow establishment. The network media visual communication design is realized through the five process stages of data: analysis, definition, integration, visual presentation, and rendering. Based on the optimal control of visual communication design, the evaluation of visual communication realization and the dissemination of visual communication information are further analyzed, and the following three conclusions are drawn: firstly, each factor does not exist alone; they influence each other. The system digs out the association rules of different factors that affect the visual communication design under the standard of $10 \%$ support and $85 \%$ confidence; secondly, comparison of the output value of the system with the real output value of the object is found that they are very close, which shows that the dynamic neural programming method is more complex and nonlinear. Finally, through the simulation of the relationship between the continuous dissemination of information and the number of tourists, on the first day, there was the first burst of new customers. The first outbreak occurred on the fifth day of the first week. In the first month, as the number of tourists increased, the dissemination of tourist information by tourists also gradually increased. This fully shows that there is always a long-term relationship between information dissemination and audiences.
\end{abstract}

\section{Introduction}

The visual communication design of network media aims to reproduce conceptual and invisible information through network carriers, and its process is generally described as a "visualization" process [1]. At the beginning of the twentieth century, visual communication has quietly entered our lives, flooding all corners of our lives. At that time, it was spread through traditional paper media such as magazines, newspapers, and posters. In the late twentieth century, with the development of digitization and the Internet, the visual communication design with digital information technology as the main medium and network communication as the main communication medium led people to a new visual direction [2]. At present, under the continuous development and changes of the market economy, the visual expression and design style changes in the network media environment are becoming more and more prominent. This has also caused some scholars to give special attention to the visual communication form and style of the network media. $s$ concern. However, excessive pursuit of the visual communication form and experience of online media also limits the new development of online media.

As data gradually enters the field of visual communication design as information, more designers have begun to rationally study the relationship between visual communication and network media and in-depth discussions on the in-depth functions and implementation methods of data- 
driven design in visual. Adding data as semantic symbols to the construction of the visual system provides a new level and possibility for visual communication. The generative design provides a path for the visual system to control the characteristics of the data. Its characteristics are reflected in the inerrability of the design process and the design results and the scalability of the design results. The dynamic and variability of data are combined with the path of generative design to form a circular path in which data can intervene, which means that every data update can bring a new visual presentation.

The huge amount of data in the design of network media visual communication, the diversified, dynamic, and realtime nature of data information put forward the requirements of a repeatable and inerrable design framework for visual reproduction. This means that the design process needs to change from the current linear static design process to nonlinear dynamic neural design process transformation [3]. Based on "data-driven," this paper explores the realization of network media visual communication design system based on nonlinear dynamic neural programming.

\section{Related Works}

With the continuous development of the Internet, network media is called the "fifth media" [4], and visual communication design has become an important research part of network media. In the past few decades, the research of visual communication has developed rapidly. Haotian, $\mathrm{Wu}$, and Li Guangan deeply analyzed the definition of visual communication and the historical evolution process and provided a favourable theoretical basis for studying the unification of the future development of design communication in the era of network media [5]. Liu, Xiang et al. mentioned that designers should not only pay attention to the technology of network media but also analyze a large number of design expressions of art and the details and concepts that design works need to have in the network media environment and combine with practice to make appropriate adjustments [6]. When appreciating successful design works, you should carefully appreciate the details of each part of the work and then combine the background analysis at the time of creation to find the source of the visual communication design performance and the characteristics that need to be summarized [7]. Wu, Haotian, and Guangan Li talked about the "occurrence and evolution of aesthetics in reconstruction," especially in the section of the reconstruction effect of art communication, how to understand the popularization of digital media aesthetics in new media, and the derivation and misunderstanding of aesthetics [8].

As early as 1960, the British art theorist EH Gombrich put forward the concept of visual thinking in "Art \& Illusion". Yang, Chao-Ming, and Tzu-Fan Hsu mentioned the in-depth discussion of "information visualization" and proposed that "such a development situation, visual communication design, has gone far beyond the traditional meaning of design. It is not just a language in the sense of traditional design, but also a comprehensive description of the comprehensive cultural phenomenon that exists in contemporary society. It is a new manifestation of the progress of social civilization" [9]. Chen, Huijuan, and Xintao Zheng professionally interpreted the works of related celebrities, the connotation of network media, the use of network media communication technology, and how to communicate in the article [10]. Krause, Kelly traced the development process of network media. Both the current development of new media and the future trend of new media have been professionally explained [11]. Huang, Min, and Dandan Luo et al. put forward: "A great advantage of vision is not only that it is a highly clear medium, but also that this medium will provide endless and rich information about various objects and events in the external world" [12]. Jiang, Bian, and He Qing et al. proposed: no matter what kind of visual design, it is particularly important to focus on effectively conveying the information you want to express to attract the public's attention [13]. Nakilcioğlu, İsmail Hakkı pointed out that "the so-called visual perception is also visual thinking” [14]. Xin, Tao, and Han You Ye explained: vision is a basic tool (or medium) of thinking, and the perceptual characteristics of visual thinking are not limited to the scope of direct perception. Perception in a broad sense also includes mental images and the connection between these images and direct perceptual grasp [15].

Due to the rapid development of information technology, it is widely used in digital art, such as web pages, character animations, and interactive games. The interactive function realizes the innovation of the passive role of the audience in the traditional art process, and the audience can also participate in the art creation process, enabling the audience to make independent judgments and choices. The process and outcome of different choices are also different, which improves the audience's participation and satisfaction. As big data began to rise and be used by people, many scholars have begun to introduce driving data into the realization of network media visual communication design. Dur, Banu Inanc Uyan et al. proposed that the field of visual communication is facing challenges in the current context. The impact and challenge come from the content that needs to be converted and reproduced into data information. The uncertainty of data structure, real-time nature, and the huge amount of information brought by big data all pose challenges for visual reproduction methods [16]. Russmann, Uta, and Jakob Svensson et al. analyzed: from graphic design, to visual communication design, to information communication, the research is gradually transformed from the reproduction-based medium to the recognition of the communication problem. However, the digitization of information types has an impact on the research of visual communication design. The research path of communication methods poses further challenges [17]. In a research by Trumbo, Jean et al., although visual representation is an important link in the field of data visualization, it is still difficult for visual communication design to establish an academic research culture in this field without understanding data [18]. Agrawala, Maneesh, Wilmot Li, and Floraine Berthouzoz discussed practical cases of lack of tradition in a study in the field of visual communication design [19]. 
In summary, the traditional realization methods for visual communication design can no longer meet the current development needs. With the development of the Internet and big data, the development of digital normalization of visual design performance in the network media environment is becoming more and more common. The receiver's single output of visual information can no longer meet the receiver's demand for information, and the data are reproduced. Dynamic and real-time nature of one of the information also calls for the emergence of new design ideas. Nonlinear dynamic programming methods can effectively compensate for the forecast errors caused by missing data and insufficient data. With the goal of minimizing the prediction error, the hyperparameter optimization of the nonlinear model has a high convergence speed and can also solve the nonlinear complex problems and achieve the best simulation and prediction accuracy. Based on the new thinking of "data-driven," this paper applies the nonlinear dynamic programming method to the realization process of network media visual communication design.

\section{Realization of Network Media Visual Communication Design}

3.1. Introducing Data-Driven Visual Communication Design. Data-driven visual communication design is a complex problem, which needs to include data generation, analysis, and reproduction of different processes, with data visualization as the core to reflect [20]. The application of datadriven thinking to the field of visual design is inspired by computer technology and programming languages, and algorithms and data are the foundation. The method of code writing quoted data to generate visual images ensures a realtime and editable working path that can realize the whole process, which is in line with data-driven design ideas, which also means that the design ideas are suitable for solving data dynamics and unpredictable features. Iterative thinking is the reproduction of complex information that provides a partial, step-by-step solution. The data is reproduced and reorganized through the code so that it can be identified according to a required logical sequence. At this time, the visual communication process serves as a logical guide. The meaning of visual information for the receiver comes from the visual representation of the "pedigree" between the information, that is, the logical relationship between the information. The visual reproducer locates meaningful information by combing and recognizing the logical relationship.

The code provides a new method and path for processing data and information. Designers can establish interpretation rules according to each specific context and generate dynamic solutions with data. At this time, the data becomes a kind of meaning representation, generating graphics with a conveying purpose. For example, the design in Figure 1 uses the receiver to trigger certain data feedback as part of the information content, expressing richer meanings, adding semantic functions to images through data, and creating a large number of data-based visual communication systems for complex information.
3.2. Data-Driven Realization of Visual Communication Design Process. When it comes to the application of data and code in the field of visual design, one has to mention the field of visualization that serves the reproduction of data and information. Data visualization is the best embodiment of the characteristics of visually reproducing data. Data visualization involves the process of visually reproducing the relationship between data and information, which is subdivided into knowledge visualization and information visualization according to different reproducing motivations: information visualization serves the observation results, and knowledge visualization conveys concepts through visual logic. The process in which one object uses another object to show its meaning is called reproduction, and the process of visually logically reproducing data or other obscure concepts is the reproduction of diagrams.

The complexity of the relationship between data determines the difficulty of the presentation of the data visualization model. The data is reproduced as content as graphics. As shown in Figure 2, it undergoes three main stages of definition, integration, and visual presentation, namely, the sorting of data and information and semantic definition. And the three steps of semantic visual transformation: using icons as a semantic carrier to express meaning, the number of the same icons reproduces the quantitative relationship from the perceptual level. After the chart framework is established, the changes of data are adjusted and changed within the framework as content, and its connotative relationships are presented with the help of a visual framework, but the data as the content itself cannot directly change the framework. Visualizing data, whether the ultimate goal is to transfer knowledge or assist in observing data, is essentially the process of establishing a visual representation framework into which data can be imported. Data-driven visual communication design ideas are embodied in two aspects: semantic framework and visual representation of data the establishment of rules. The establishment of a semantic framework is embodied in the process of defining and integrating key data variables. This process involves the extraction of meaningful data variables and the definition of meaning; the visual presentation of data relationships is to reduce the impact on the data through visual aids. The basic principle of the difficulty of observing the change trend is to reproduce the meaning of the data change through the perceptual potential of the image.

In summary, the application of data-driven visual communication design ideas to the field of visual communication design can provide ideas for establishing a flexible visual system based on data variables through the definition of semantic framework and visual reproduction rules, so as to cooperate with the dynamics and richness of data.

3.3. The Process of Establishing a Visual System Driven by Network Media Data. Figure 3 shows the definition and integration of the entire generative design path from the visual prototype. The visual prototype is the starting point and the end point of the final visual solution output. It is a 


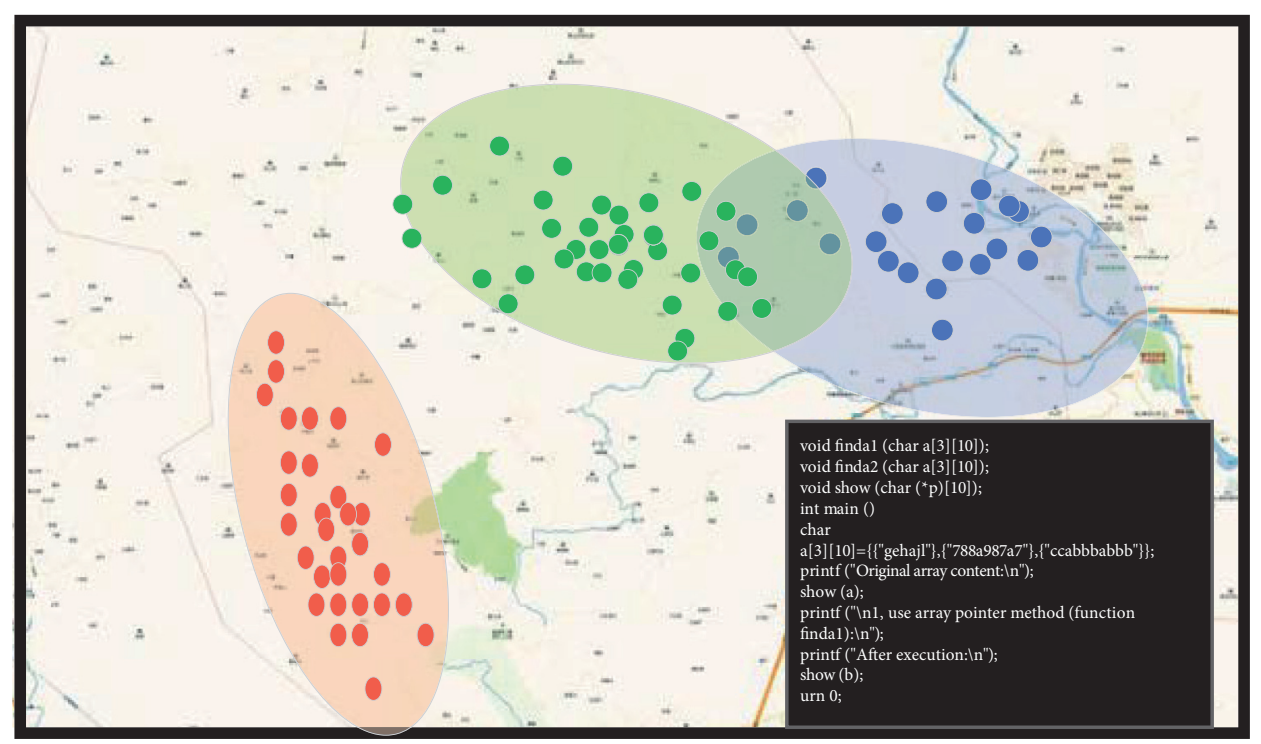

FIgURE 1: Visual works based on data.

process of constant trial and error feedback. After the visual prototype is established, the available data is introduced. There is no direct relationship between the visual prototype and the available data at this time. The data is organized, and the exploration is carried out in the second stage. Define meaningful variables, such as wind speed, wind direction, and so on in the above example; make a one-to-one correspondence with visual elements; and confirm that visual elements can perform data model characteristics in a certain perceptual potential direction. The corresponding process of visual elements and data is author-oriented. The only criterion is whether visual elements can use the perceptual potential of the elements to reproduce the changing trend of data. If the number of meaningful variables does not match the number or nature of the visual elements that can be represented, the workflow will go back to the first step, remodify the visual reproduction scheme, and enter the above-mentioned process again. Matching is based on the performance data characteristics of another visual element under the visual element. For example, in the above example, the depth of the red colour of the rectangular colour block in the picture represents the temperature. The integration step evaluates the overall visual logic based on the correspondence between the data and the visual representation. If the visual process or the visual symbol at the same level points to a wrong variable relationship, you need to return to the visual representation scheme and semantic definition stage to evaluate again.

\subsection{Research on Visual Communication Methods of Network Media}

3.4.1. Data-Driven Nonlinear Dynamic Programming Method. Based on the data-driven nonlinear dynamic programming method, the programming problem with the objective function or the nonlinear function of the independent variable contained in the constraint condition belongs to the nonlinear programming. Nonlinear programming can be divided into unconstrained nonlinear programming and constrained nonlinear programming. The general solutions for unconstrained nonlinear programming include the steepest descent method, conjugate direction method, and Newton's method. The general solutions for constrained nonlinear programming include the gradient projection method, reduced gradient method, penalty function, and barrier function method. Nonlinear programming can solve many problems such as the optimization of nonlinear functions, complex logistics, and transportation problems. This paper uses an improved nonlinear neural algorithm to effectively improve the problem of slow system convergence. The algorithm in this paper has become one of the important models of neural networks, laying a theoretical foundation for the correct design of network media visual communication design systems. We use nonlinear programming to solve the problem of discrimination of dynamic data.

Definition 1. Suppose we divide a continuous attribute data that needs to be discredited into three categories and give two segmentation points by the method of equal-width discrimination. With a given degree of overlap, we can get a fuzzy set on the attribute.

$$
\begin{aligned}
& a=(1+\partial) p^{1}-\partial l, b=p^{1}-\partial\left(p^{2}+p^{1}\right) * 2, \\
& c=p^{2}+\partial\left(p^{2}+p^{1}\right) * 2, d=(1+\partial) p^{2}-\partial u
\end{aligned}
$$

The generation of these fuzzy sets can be further described as follows: 


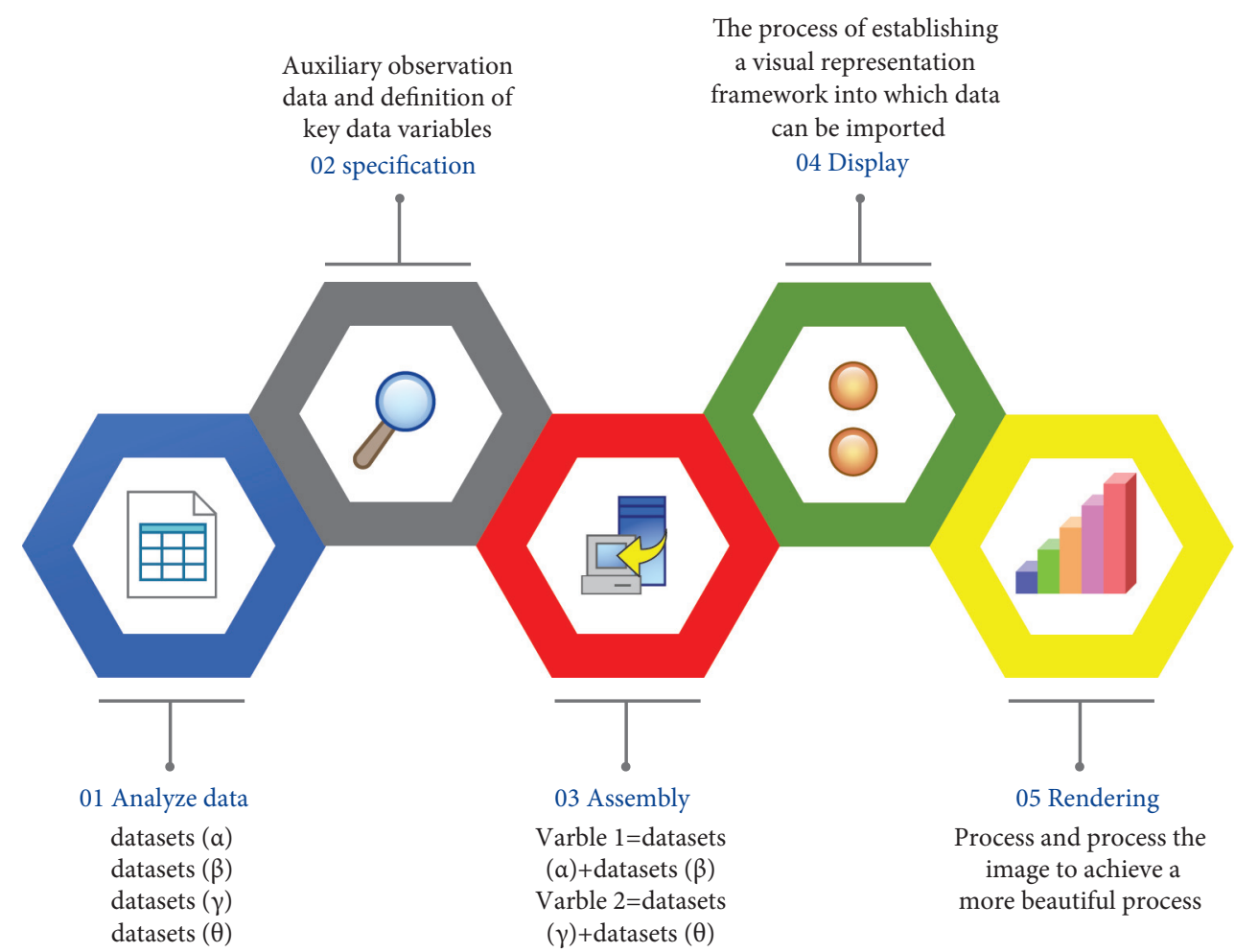

FIGURE 2: Data visualization workflow.

$$
\begin{aligned}
& f_{1}(x)= \begin{cases}1, & 1 \leq x \leq a, \\
\frac{x}{a+b}-\frac{c}{b+a}, & a \leq x \leq b,\end{cases} \\
& f_{2}(x)= \begin{cases}\frac{x}{a+b}-\frac{a}{b+a}, & a \leq x \leq b, \\
1, & b \leq x \leq c,\end{cases} \\
& f_{3}(x)= \begin{cases}\frac{x}{c+d}-\frac{b}{d+c}, & c \leq x \leq d, \\
\frac{x}{d+c}-\frac{c}{c+d}, & c \leq x \leq d .\end{cases}
\end{aligned}
$$

Definition 2. Fuzzy $C$-means clustering (hereinafter referred to as FCM) is a method for fuzzy division of a group of data. Under the premise of a given number of division groups, this method can automatically determine the center points of each group and give the degree of membership of each data relative to each group. This algorithm is based on minimizing the following functions:

$$
Q_{m}=\sum_{i=1}^{N} \sum_{J=1}^{C} u_{i j}^{m}\left\|x_{j}+c_{j}\right\|^{2}, \quad 1 \leq m \leq \infty,
$$

where $u_{i j}$ is the degree of membership of data $x_{\mathrm{i}}$ relative to group $j, c_{j}$ is the center point of each group, and $\|\cdot\|$ is an operator that indicates the degree of similarity between data. The FCM classic algorithm is to find the minimum value of this function through iterative optimization, so as to obtain the $u_{i j}$ and $c_{j}$ under the optimal conditions. The specific algorithm is as follows:

(i) Select the initial matrix $U=\left[u_{i j}\right]$, denoted as $U_{(0)}$

(ii) In the $k$-th step: calculate the center point $C_{(k)}=\left[c_{j}\right]$ through $U_{(k)}$ as follows:

$$
c_{j}=\frac{\sum_{J=1}^{C} u_{i j}^{m}-x_{i}}{\sum_{J=1}^{N} u_{i j}^{m}} .
$$

(iii) Upgrade to $U_{(k+1)}$

$$
u_{i j}=\frac{1}{\sum_{J=1}^{N}\left[\left\|x_{i}+c_{j} /\right\| x_{i}+c_{k}\right](2 / m+1)} .
$$

(iv) If as shown in formula (6), go back to step 2.

$$
\left\|u^{(k-1)}+U^{(K)}\right\|>\prec \leq \beta .
$$

3.4.2. Data-driven Nonlinear Dynamic Programming Algorithm Implementation. The FCM algorithm is essentially a local search optimization method. Its iterative process uses a so-called hill-climbing method to find the optimal 


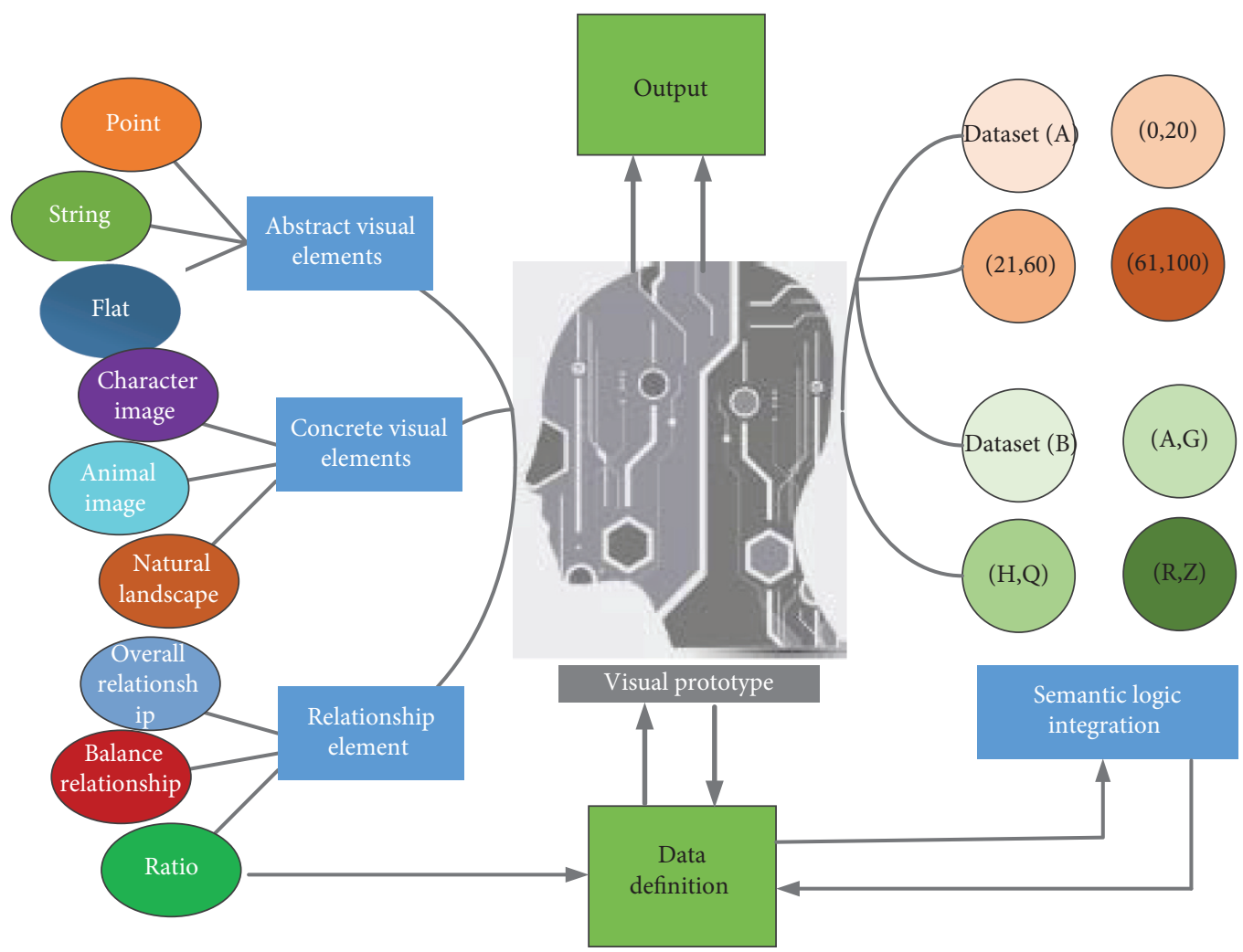

Figure 3: Data-driven vision system establishment process.

solution. Therefore, the algorithm is very easy to fall into a local minimum and cannot get the global optima. Since the essence of FCM is an optimization problem, we built a nonlinear programming mathematical model based on this, trying to avoid the drawbacks of the classic algorithm and directly find the global optimal solution:

$$
c_{j=} \frac{\sum_{J=1}^{C} u_{i j}-x_{i}}{\sum_{J=1}^{N} u_{i j}} .
$$

(i) Select the initial center value $C=\left[c_{j}\right]$, denote it as $C_{(0)}$

(ii) In step $k$ : bring $C_{(k-1)}$ into the following model to solve $U_{(k)}$ :

$$
\begin{aligned}
\min J & =\sum_{i=1}^{n} \sum_{J=1}^{n} u_{i j}^{m}\left\|x_{j}+c_{j}\right\|^{2}, \quad 0 \leq u_{j} \leq 1, \\
\min J & =\sum_{i=1}^{n} \sum_{J=1}^{n} u_{i j}^{m}\left\|x_{j}+c_{j}\right\|^{2}, \quad 0 \leq u_{j} \leq 1, \\
0 & \leq \sum_{J=1}^{n} u_{i j}^{m} \leq n, \\
\sum_{J=1}^{n} u_{i j}^{m} & =1 .
\end{aligned}
$$

(iii) Calculate the center point $C_{(k)}=\left[c_{j}\right]$ through $U_{(k)}$ :

$$
c_{j=} \frac{\sum_{J=1}^{C} u_{i j}-x_{i}}{\sum_{J=1}^{N} u_{i j}} .
$$

(iv) If it is as in formula (10), stop; otherwise, return to step 2:

$$
\left\|C^{(k-1)}+C^{(K)}\right\|>\prec \leq \beta .
$$

Although this algorithm also needs to be iterated and may fall into a local optimal solution, it uses a nonlinear programming method when calculating $U_{(k)}$. Therefore, it is better than the FCM classic algorithm in terms of the number of iterations and the optimality of the solution. The improvement also solves the problem of the initial value of $c_{j}$, which can be realized by programming with GAMS software.

3.4.3. Using Nonlinear Dynamic Programming Method to Calculate. For example, 10 integers are divided into 3 categories, and the 10 numbers are as follows: 112, 20, 3,300, $4,200,52,623,745,8,120,93$, and 10,344; the initial value of the 3 central values is 8 ; after running the GAMS program, the results are as follows:

(i) The first category includes: 23,45 , and 120 , with a center value of 62.667 
(ii) The second category includes: $12,0,2$, and 3 , with a center value of 4.250

(iii) The third category includes: 300,200 , and 344, the center value of 281.333 (a total of 3 iterations)

In order to further illustrate the effect of this method, we will use this method to discretize the actual data and apply the discretization results to the analysis of actual problems.

In summary, in the current technical context, data has become an information object that needs to be considered in the field of visual communication design. This chapter focuses on the realization of visual communication design based on data-driven, introduces data-driven visual communication design process realization and visual communication design process from visual communication design, and applies the data-driven nonlinear dynamic programming method to the vision of network media. In the communication design process, the next step is to further apply and analyze the network media visual communication design based on the data-driven nonlinear dynamic programming method. The FCM algorithm is essentially a local search optimization method. Its iterative process uses a socalled hill-climbing method to find the optimal solution. Therefore, the algorithm is very easy to fall into a local minimum and cannot get the global optimal. Since the essence of FCM is an optimization problem, we built a nonlinear programming mathematical model based on this, trying to avoid the drawbacks of the classic algorithm and directly find the global optimal solution.

\section{Application and Analysis of Network Media Visual Communication Design}

4.1. Analysis of Audience Evaluation of Visual Communication Design. Combined with an example, our data set comes from the relevant information collected after a questionnaire survey of 5,000 users of a certain online media within 1 month. Due to a large amount of information, we selected part of the questionnaires to use our nonlinear dynamic programming method for analysis and research. First of all, the originally designed questionnaire is quite detailed, involving all aspects of visual communication. It analyzes the association rules of more than 40 attributes, which requires a large amount of data to obtain more accurate association analysis results, but the interpretability of these obtained association rules may be challenged. Of course, another problem is that our current solutions cannot solve the discretization of relatively large-scale data. Therefore, we use the statistical software SAS to perform factor analysis on the data set and synthesize all the questionnaire questions (attributes) into 10 factors, representing the audience's evaluation in ten aspects: Factor $1=$ Information, Factor $2=$ Colour, Factor $3=$ Text,$\quad$ Factor $4=$ Image,$\quad$ Factor $5=$ Convenience, Factor6 $=$ Audio, Factor7 $=$ Layout, Factor $8=$ Interaction, Factor $9=$ Video, and Factor $10=$ Layout. At the same time, calculate the value of each factor for each audience. The higher the factor value, the higher the customer's satisfaction in this respect and the higher the evaluation as shown in Figure 4.

Finally, we use Weka software to discretize the data and then conduct association rule mining. Under the standard of $10 \%$ support and $85 \%$ confidence, the following results are obtained:

(i) Factor $4=2$ Factor $6=310$ Factor $1=210$, that is, an audience who is satisfied with text and images and is satisfied with the attractiveness of the information

(ii) Factor $4=2$ Factor6 $=3$ Factor7 $=38$ Factor $1=28$, that is, audiences who are satisfied with the text and images but are not satisfied with the audio are also satisfied with the attractiveness

(iii) Factor $1=2$ Factor $4=2$ Factor5 $=28$ Factor7 $=38$, that is, they are satisfied with the colour and interactivity, and the audience who think the layout design is generally dissatisfied with the text information

(iv) Factor $4=2$ Factor5 $=2$ Factor7 $=39$ Factor $1=28$, that is, they are satisfied with the service but dissatisfied with the convenience, and audience who think that the layout design is generally dissatisfied with the interactivity

The above is the association rules mined under the standard of support of $10 \%$ and confidence of $85 \%$. The support and confidence are adjusted as needed, and different association rules will be mined.

\subsection{Optimal Control Analysis of Visual Communication} Design. The nonlinear neural network method aims at minimizing the prediction error, has a high convergence speed, and can reach the best simulation and prediction accuracy. This summary compares the prediction accuracy and error of the nonlinear neural network method and the traditional BP algorithm through experiments.

The red in Figure 5 is the output of the identified object, and the yellow is the output of the nonlinear neural network model. It can be seen that the output value of the system after the nonlinear neural network is very close to the real output value of the identified object. The nonlinear neural network model has a good ability to identify more complex nonlinear object models. It can summarize the relationship implicit in the input/output data of the system so that it can be used when the real object model of the system is unknown. The nonlinear neural network model obtains the data output of the system and completes the system identification.

Figure 6 shows the error curve between the output of the nonlinear neural network and the output of the identified object. The upper part represents the error curve of the ordinary $\mathrm{BP}$ algorithm, and the lower part represents the error curve of the nonlinear programming method. It can be seen that after the nonlinear programming method is adopted, the system error convergence speed is significantly improved, indicating that the nonlinear programming method is adopted. The system can reduce the error in a short time. 


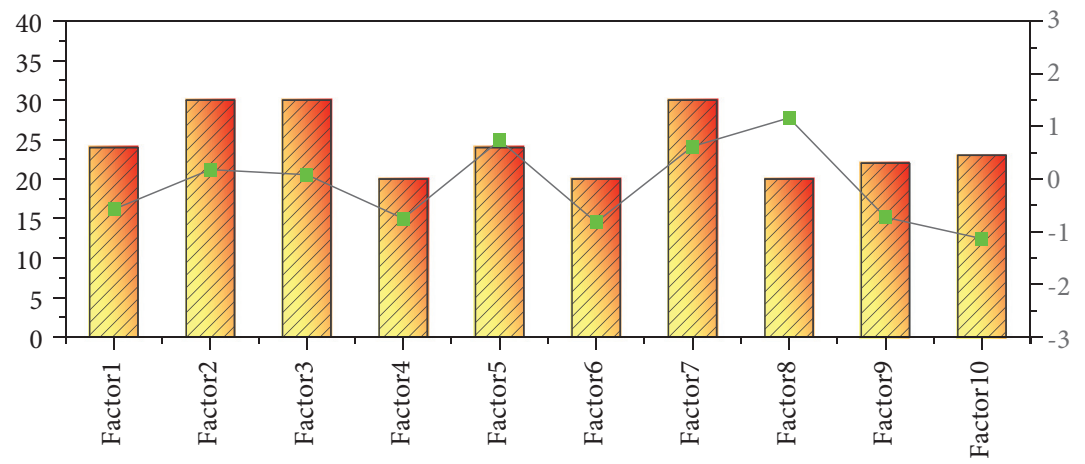

7 Number of first type - - Center value of the first type

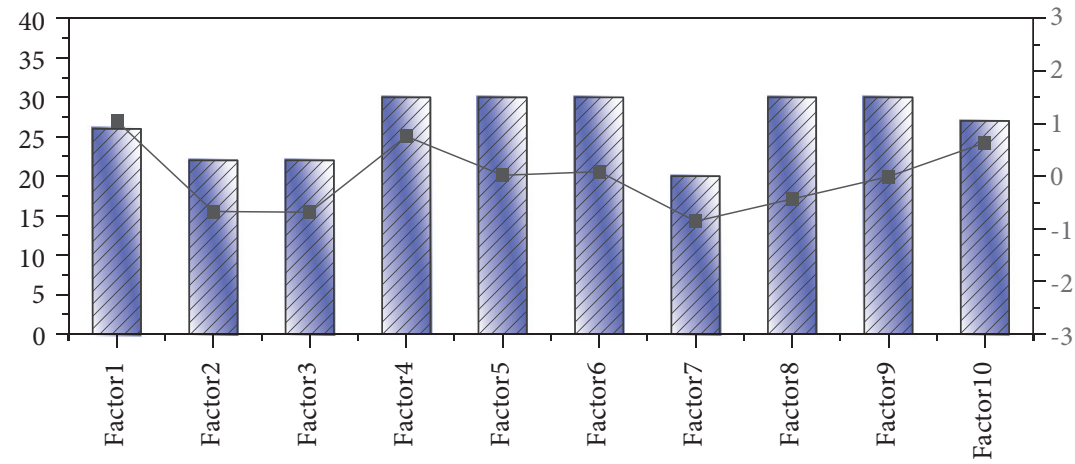

Number of second type

- Center value of the second type

FIGURE 4: Various types of factors and median value statistics chart.

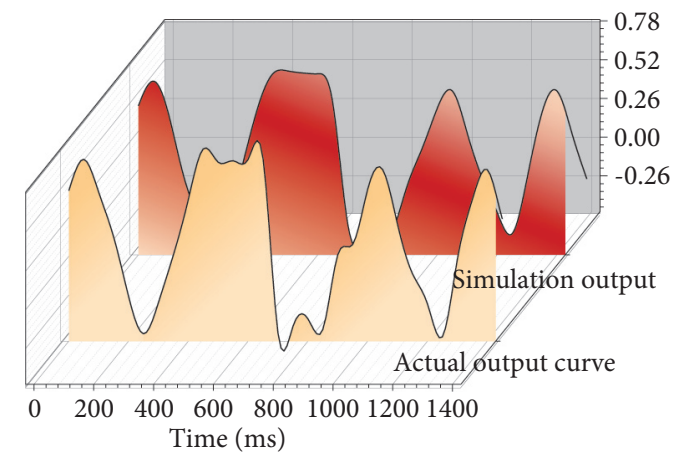

FIGURE 5: Graph of nonlinear output value and true output value.

4.3. Visual Communication Design Information Dissemination Analysis. The purpose of visual communication is to realize the process of information dissemination. Through the analysis of tourism promotion information released by a certain network media, because there are many active Internet users during the day, because the interpersonal communication in new media needs to be "online", it is usually a message the dissemination is completed within a day. However, in real life, in order to promote publicity, tourist destinations usually carry out multiple information promotions, resulting in the continuous dissemination of information. In addition, due to holidays, weather, and other reasons, there is a certain time lag between travel behaviour and information dissemination. In this chapter, through a 1- year study of 3 tourist destinations in 2020, we find the relationship between the continuity of information and the number of tourists.

As shown in Figure 7, the relationship between the continuity of information and the number of visitors was simulated. The duration of information dissemination was 1 day, 1 week, and January: on the first day, within 8-10 hours, the first burst of new customers occurred and then gradually stabilized; the first outbreak occurred on the fifth day of the first week. As tourism activities are affected by people's free time, there is a time lag between the number of tourists and the dissemination of tourism information, so the tourists in the tourist destination will also be affected, and the tourism volume will explode. The amount of tourism broke out on 


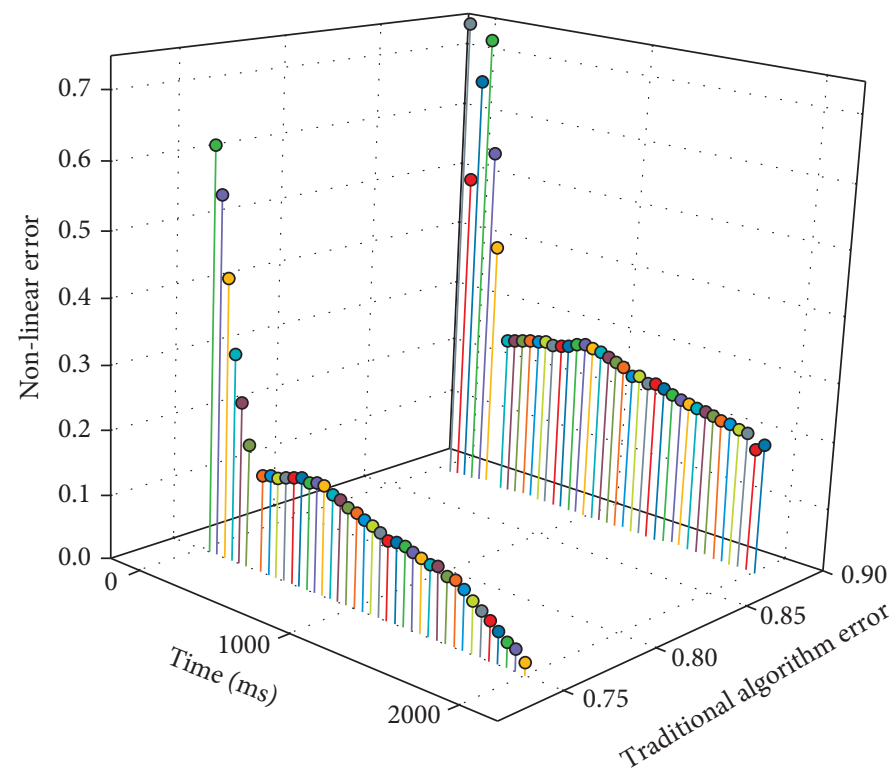

Figure 6: Nonlinear output error curve.

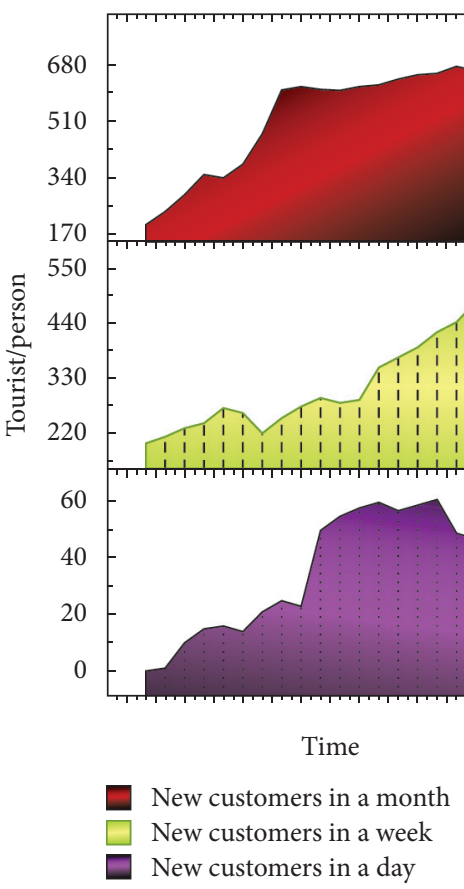

Figure 7: Diagram of the relationship between propagation time and visitor volume.

the sixth and seventh days; in the first month, as the number of tourists increased; the dissemination of tourist information by tourists also gradually increased so that the amount of information dissemination in tourist destinations was maintained at a high level, forming a continuous tourism information break out. Continued tourism information once again caused an increase in the number of tourists, so the second week again triggered an increase in the number of tourists. This shows that there is always a long-term relationship between the dissemination of tourism information and the number of tourists.

\section{Conclusion}

In this paper, data are involved in the path of network media visual design, and nonlinear dynamic neural programming methods are used to construct a network media visual communication design system from the three aspects of visual communication design: data-driven, visual communication design process realization, and visual system establishment process. Combined with examples, this paper further analyses and studies the optimal control design, implementation, and evaluation of visual communication in network media. The conclusion of visual communication information is that the data-driven system can mine the association rules between different factors affecting visual communication design and draw a conclusion. In the process of visual communication design, the implicit relationship summarizes the interaction between visual in the input/output data of the system communication information dissemination and time. This article briefly discusses the application of visual communication design and data driven in the field of network media. How to apply it to other fields is worthy of our further exploration. I believe that in the future, with the continuous development of computer technology and internet technology, data-driven applications in the field of network media and visual communication design will be broader.

\section{Data Availability}

The data used to support the findings of this study are available from the corresponding author upon request.

\section{Conflicts of Interest}

The authors declare that they have no known competing financial interests or personal relationships that could have appeared to influence the work reported in this paper. 


\section{Acknowledgments}

This paper was supported by Hunan Social Science Fund Project (19YBA105), Rural Tourism Specialty Product Packaging Driven by Rural Revitalization Strategy.

\section{References}

[1] Y. Zhou, X. Hu, and M. Shabaz, "Application and innovation of digital media technology in visual design," International Journal of System Assurance Engineering and Management, vol. 20, no. 13, pp. 5-11, 2021.

[2] A. Azadeh, F. Shafiee, R. Yazdanparast, J. Heydari, and A. Keshvarparast, "Optimum integrated design of crude oil supply chain by a unique mixed integer nonlinear programming model," Industrial \& Engineering Chemistry Research, vol. 56, no. 19, pp. 5734-5746, 2017.

[3] W. Andriyan and V. Anesti, "Visual audio communication design on the role of information technology on student life style of universitas raharja," ADI Journal on Recent Innovation, vol. 2, no. 3, pp. 15-24, 2020.

[4] Q. Zhao, "The application of augmented reality visual communication in network teaching," International Journal of Emerging Technologies in Learning, vol. 13, no. 7, pp. 50-64, 2018.

[5] Wu Haotian and G. Li, "Innovation and improvement of visual communication design of mobile app based on social network interaction interface design," Multimedia Tools and Applications, vol. 79, no. 5, pp. 23-36, 2020.

[6] X. Liu, "Application of the Internet technology in the visual communication design," in Proceedings of the International conference on Big Data Analytics for Cyber-Physical-Systems, pp. 374-376, Springer, Singapore, January 2020.

[7] M. Schreiber, "Showing/sharing: analysing visual communication from a praxeological perspective," Media and Communication, vol. 5, no. 8, pp. 37-50, 2017.

[8] H. Wu and G. Li, "Visual communication design elements of Internet of Things based on cloud computing applied in graffiti art schema," Soft Computing, vol. 24, no. 11, pp. 8077-8086, 2020.

[9] C.-M. Yang and T.-F. Hsu, "New perspective on visual communication design education: an empirical study of applying narrative theory to graphic design courses," International Journal of Higher Education, vol. 6, no. 2, pp. 188-198, 2017.

[10] H. Chen and X. Zheng, "Application of traditional culture based on computer technology in modern visual communication design," Journal of Physics: Conference Series, vol. 1744, no. 3, pp. 104-113, 2020.

[11] K. Krause, "A framework for visual communication at $\mathrm{Na}$ ture," Public Understanding of Science (Bristol, England), vol. 26, no. 6, pp. 15-24, 2017.

[12] M. Huang and D. Luo, "Research on the application Strategy of mixed teaching mode of visual communication design specialty in colleges and universities based on multidimensional interaction," E3S Web of Conferences, vol. 251, no. 3, pp. 91-112, 2021.

[13] B. Jiang and Q. He, "Research on the innovation of multimedia technology and visual communication technology," Journal of Physics: Conference Series, vol. 1693, no. 56, pp. 1628-1641, 2020.

[14] Nakilcioğlu and H. İsmail, "The effects of font type choosing on visual perception and visual communication," Online Journal of Art and Design, vol. 1, no. 3, pp. 35-53, 2017.
[15] T. Xin and Y. Ye Han, "The application of visual illusion in the visual communication design," IOP Conference Series: Materials Science and Engineering, vol. 317, no. 15, pp. 296-297, 2018.

[16] B. I. U. Dur, "Data visualization and infographics in visual communication design education at the age of information," Journal of Arts and Humanities, vol. 3, no. 5, pp. 39-50, 2016.

[17] U. Russmann and J. Svensson, "Introduction to visual communication in the age of social media: conceptual, theoretical and methodological challenges," Media and Communication, vol. 5, no. 4, pp. 24-32, 2017.

[18] J. Trumbo, "Essay: seeing science: research opportunities in the visual communication of science," Science Communication, vol. 21, no. 4, pp. 379-391, 2016.

[19] M. Agrawala, W. Li, and F. Berthouzoz, "Design principles for visual communication," Communications of the ACM, vol. 54, no. 4, pp. 60-69, 2020.

[20] N. W. Kim, "Data-driven guides: supporting expressive design for information graphics," IEEE Transactions on Visualization and Computer Graphics, vol. 23, no. 21, pp. 491-500, 2016. 